\title{
Use of Zidovudine and Interferon Alpha with Chemotherapy Improves Survival in both Acute and Lymphoma Subtypes of Adult T-cell Leukaemia/Lymphoma
}

Andrew Hodson ${ }^{1}$, Siobhan Crichton ${ }^{2}$, Silvia Montoto ${ }^{3}$, Naheed Mir $^{4}$, Estella Matutes ${ }^{5}$, Kate Cwynarski ${ }^{6}$, Thurai Kumaran ${ }^{7}$, Kirit Ardeshna ${ }^{8}$, Antonio Pagliuca ${ }^{9}$, Graham P Taylor $^{1,10}$ and Paul Fields ${ }^{11}$.

\footnotetext{
${ }^{1}$ National Centre for Human Retrovirology, St Mary's Hospital, Imperial College Healthcare NHS Trust, London.

${ }^{2}$ King's College London, United Kingdom.

${ }^{3}$ Barts Cancer Institute, Queen Mary University of London, London, United Kingdom.

${ }^{4}$ University Hospital Lewisham NHS Trust, London, United Kingdom.

${ }^{5}$ The Royal Marsden NHS Foundation Trust, London, United Kingdom.

${ }^{6}$ The Royal Free Hampstead NHS Trust, London, United Kingdom.

${ }^{7}$ North Middlesex University Hospital, London, United Kingdom.

${ }^{8}$ University College Hospital NHS Foundation Trust, London, United Kingdom.

${ }^{9}$ King's College Hospital, London, United Kingdom.

${ }^{10}$ Imperial College, London, United Kingdom.

${ }^{11}$ Guy's and St Thomas' NHS Foundation Trust, London, United Kingdom.
}

Corresponding author: Dr Paul Fields, MD, PhD, Department of Haematology, Guy's and St Thomas' NHS Foundation Trust, Great Maze Pond, London, SE1 9RT, United Kingdom; telephone: 0207188 0141; fax: 0207188 2748; e-mail: paul.fields@gstt.nhs.uk

Presented in part at the 2010 Annual Congress of the American Society of Haematology, Orlando, FL, December 4-7, 2010.

Disclosures - the authors indicate no potential conflicts of interest 


\section{Abstract}

Purpose

Adult T cell leukaemia/lymphoma (ATLL) is a mature (post-thymic) T cell lymphoma associated with human T-lymphotropic virus type 1 (HTLV-1) infection. Survival in aggressive subtypes remains poor and treatment resistance is frequent. Use of zidovudine (ZDV) and interferon-alpha (IFNa) has been associated with improved response rates in small studies and prolonged overall survival in leukaemic ATLL subtypes in a recent meta-analysis.

Patients and methods

We report the clinico-pathologic characteristics, treatment and outcome of 73 patients with aggressive ATLL (29 acute ATLL; 44 lymphoma ATLL) diagnosed and treated in England between 1999 and 2009. The impact of ZDV/IFNa on treatment response and survival was assessed.

Results

The overall response rate ranged from $55 \%$ with chemotherapy alone to $81 \%$ with combined first line therapy (chemotherapy with concurrent/sequential ZDV/IFNa). Median overall survival (OS) was 9 months: 7.5 months for acute ATLL and 10 months for lymphoma ATLL. Use of ZDV/IFNa at any time prolonged survival in acute $(p=0.0007)$ and lymphoma ATLL $(p=0.0004)$ and was the sole factor associated with reduction in risk of death in aggressive ATLL (hazard ratio 0.23, 95\% $\mathrm{Cl}$ 0.09-0.60, $\mathrm{p}=0.002$ ). Combined first line therapy prolonged median $\mathrm{OS}$ in acute $(p=0.0081)$ and lymphoma ATLL $(p=0.001)$ compared with chemotherapy alone.

\section{Conclusion}

These data support the use of low dose ZDV/IFNa with chemotherapy in the first line treatment of acute and lymphoma ATLL. 


\section{Introduction}

Adult T-cell leukaemia/lymphoma (ATLL) is an aggressive T-cell non-Hodgkin's lymphoma (NHL) initially described in 1977 (1) and soon after demonstrated to be associated with the retrovirus human T-cell lymphotropic virus type 1 (HTLV-1) (2). HTLV-1 is endemic in the Caribbean basin, parts of Central and South America, Central Africa, regions of Japan and Iran. In the United Kingdom it is estimated that 22,500 people are infected with HTLV-1 and infection is most frequent in individuals of Afro-Caribbean or African origin (3). HTLV-1 is frequently transmitted from mother to child via breast feeding and several strands of evidence point to the importance of early childhood infection in the development of ATLL: the prolonged period between infection and presentation with disease and the high prevalence of HTLV-1 infection in mothers of patients with ATLL compared with other HTLV-1-associated diseases (4). Between $3-5 \%$ of individuals who become infected with HTLV-1 before the age of 20 will develop ATLL and risk of leukaemia has been associated with acquisition of infection in early life $(5,6)$ and with high HTLV-1 proviral load in adults $(7)$.

ATLL is classified according to the criteria of Shimoyama (8). Sub-classification into acute, lymphoma, chronic or smouldering ATLL is based on lymphocyte count, abnormal lymphocyte percentage, lactate dehydrogenase (LDH), corrected calcium and pattern of tissue involvement. Median survival of patients with acute and lymphoma ATLL was 6 and 10 months, and projected four-year survival rates 5 and $6 \%$, respectively. These two subtypes represent 'aggressive' ATLL, the median survival in patients with chronic ATLL being 24 months (7).

The reported incidence of ATLL in the United Kingdom is $14-16$ cases per annum (9). Between 1996-9 all 37 cases in the UK were in England and diagnosed in patients of Afro-Caribbean origin (3). The mean age at presentation was 40 years, similar to other series of Afro-Caribbean patients $(10,11)$ and a decade younger than in Japanese patients $(12,13)$.

ATLL was first reported in the UK in 1982 in six Afro-Caribbean patients aged 21-55 who underwent brief remissions to combination chemotherapy (14). Further UK experience is limited to two case series and two prospective studies. In both case series, including 52 and 21 patients respectively, median overall survival (OS) was 
only 6 months $(15,16)$. In 2001 a prospective study assessed the tolerability and efficacy of zidovudine (ZDV) and interferon- $\alpha$ (IFN- $\alpha$ ) in 15 subjects and compared to historical controls. Most patients had acute ATLL. Twelve had received prior chemotherapy, including one of two with smouldering ATLL. The response rate was $67 \%$ and the difference in survival between responders and non-responders was significant $(p=0.002)$ suggesting a benefit with use of ZDV and IFN- $\alpha$. The median OS from first presentation was 18 months (17). Most recently a phase 2 study assessed the addition of an anti-CD25 monoclonal antibody (Daclizumab) to first line therapy with cyclophosphamide, Adriamycin (doxorubicin), Oncovin (vincristine), prednisolone (CHOP), in 10 subjects with aggressive ATLL (18). The overall response rate was $70 \%$ with five patients entering CR, which was maintained for 24 months in two cases. However disease progression and early relapse was typical and seven patients died 4-19 months from diagnosis.

The aim of this study was to describe aggressive ATLL treatment patterns in England and compare outcomes to published data. In particular the impact of therapy with zidovudine and interferon-alpha (ZDV/IFN- $\alpha$ ) was assessed in view of the reported efficacy in ATLL $(17,19,20)$.

\section{Methods}

Subjects

We conducted a retrospective analysis of data from patients diagnosed with aggressive ATLL in England between January 1999 and December 2009.

Participating centres specialized in the treatment of aggressive lymphomas or had a relatively high incidence of ATLL. Patient data were obtained from medical records according to the study proforma and included demographic data, disease subtype, treatment, response and survival and were anonymised for analysis.

This study was approved by the Joint Research Office of Imperial College Healthcare NHS Trust and by local Research and Development offices in each major participating centre. 


\section{$\underline{\text { Definitions }}$}

Essential diagnostic criteria for ATLL were histological and/or cytological evidence of a CD4, CD25 positive T-cell malignancy (based on immunohistochemistry or flow cytometry) with abnormal T lymphocytes in the peripheral blood (except in the lymphomatous form of ATLL) and antibodies to HTLV-1. Patients were classified according to the Lymphoma Study Group (Shimoyama) criteria into acute or lymphoma ATLL (8). The term 'aggressive' refers to the acute and lymphoma subtypes grouped together.

Anti-viral treatment (AVT) refers to combination treatment with IFN- $\alpha$ and ZDV although in the absence of HTLV-1 replication in ATLL the mechanism is not thought to be directly anti-viral. Three types of treatment strategy were observed:

1. 'Chemotherapy alone' being only chemotherapy with no AVT at any time

2. 'Combined first line therapy' being AVT given concurrent with, or immediately sequential to, first line chemotherapy;

3. 'Deferred AVT' being initial chemotherapy with AVT given at any time following relapse

In some analyses the last two categories are combined and referred to as 'AVT at any time'.

\section{$\underline{\text { Response criteria }}$}

To allow comparison with previous ATLL studies response to treatment was assessed according to Japan Clinical Oncology Group criteria (21). Complete response (CR) was defined as normalization of the white blood cell count (WBC) and corrected plasma calcium concentration along with the disappearance of all measurable tumours on CT imaging. Partial response (PR) was defined as a decrease of greater than or equal to $50 \%$ in the WBC and the size of measurable tumours. All responses had to last at least one month. All other outcomes were classified as no response. 


\section{Statistical analysis}

Overall survival (OS) was measured from date of diagnosis to death or date of last patient contact if alive. Data were censored at 01/07/2010. Response rates according to treatment type were compared using Pearson's chi-square test. Patient survival data were analysed using Kaplan and Meier curves and compared using the log-rank test. To assess the impact of individual factors on survival multivariate analyses were performed using the Cox proportional hazards model.

All statistical calculations were performed using STATA 11 MP (Statacorp LP, Texas, USA).

\section{Patient characteristics}

Of the seventy-three patients 70 (96\%) were African/Afro-Caribbean. Forty-four $(60 \%)$ were female. Median age at diagnosis was 58 years (females 62, males 56). According to the Shimoyama classification 44 patients had lymphoma and 29 acute ATLL. The median ECOG performance status was 1 (range 0-4). Central nervous system (CNS) involvement comprised one patient with primary CNS ATLL and 12 patients with lymphocytes of typical ATLL morphology in cerebrospinal fluid (CSF). Patient characteristics are summarised in Table 1.

Six patients treated palliatively due to poor performance status and co-morbidities, including two given low dose AVT alone for up to 14 days, died soon after diagnosis and were not included in the treatment analyses.

\section{$\underline{\text { Results }}$}

\section{$\underline{\text { Treatment }}$}

Of the 67 patients who received chemotherapy as first line treatment 27 had acute ATLL and 40 lymphoma ATLL. In 54 patients without CNS involvement the chemotherapy regimen was CHOP alone $(n=45)$, CHOP with the anti-CD25 monoclonal antibody Daclizumab (CHOP-Z; $n=7)$ or Gemcitabine/Carboplatin $(n=2)$. Thirty-four (63\%) received prophylactic intra-thecal chemotherapy (ITC). Of the 13 with CNS involvement six received CHOP (with Daclizumab in three patients) and 
additional ITC while the other seven were treated with high dose IV methotrexate $(n=5)$ or cytarabine $(n=2)$ containing regimens.

Forty patients (60\%) were treated with 'AVT at any time': 18/27 (67\%) acute, 22/40 (55\%) lymphoma. Twenty-six patients were treated with 'combined first line therapy' (AVT concurrent with chemotherapy in 11 and sequential in 15). The remaining 14 patients received 'deferred AVT', of whom six were primary progressors on first line chemotherapy.

The recommended AVT regimen was IFN- $\alpha 3$ million units (MU) daily and ZDV $250 \mathrm{mg}$ twice daily but various doses and intervals were documented. Concurrent AVT was started with the first or second cycle of chemotherapy in 10 patients and with cycle four in the other. Where sequential, AVT was started within two months of completion of chemotherapy. The median duration of AVT was 4.5 months (3 days 77 months). Of the patients who did not receive AVT only one died before AVT could reasonably have been initiated, within one month of diagnosis.

Toxicity reporting, while incomplete, was documented in 19 out of 40 cases who received AVT. AVT was stopped in three patients for severe myelosuppression, combined cardiac failure and nephrotic syndrome (likely secondary to IFN- $\alpha$ ) and lactic acidosis respectively. Haematological toxicity was most frequent, in particular in patients treated with prior chemotherapy: Grade 3 neutropenia in three patients and grade 1/2 in eight, eight reports of anaemia (one grade 3, four grade 2) and two of thrombocytopenia (one grade 4).

The use of prophylaxis against opportunistic infection (OI) was documented in $62 \%$ of cases, and included co-trimoxazole or dapsone, aciclovir and fluconazole or itraconazole.

\section{$\underline{\text { Response to first line treatment }}$}

Treatment response data were available for $65 / 67$ patients receiving first line chemotherapy. The response rate in patients receiving 'combined first line therapy' was $81 \%$ and in those receiving chemotherapy alone, $49 \%(p<0.01$, Odds ratio 4.42) (Table 2). 
Six patients (4 CR, $1 \mathrm{PR}, 1 \mathrm{NR}$ ) proceeded to allogeneic stem cell transplantation (alloSCT), five from related donors, after first line therapy.

\section{Progression-free survival and overall survival}

Although treatment with 'combined first line therapy' led to an approximate doubling in progression free survival compared to chemotherapy alone ( $8 \vee 4$ months) this did not reach statistical significance. This observation was made in both acute ( $8 \vee 5$ months) and lymphoma (7 $\vee 4$ months).

The median OS for all 73 patients with aggressive ATLL was 9 months (range 0.379), 7.5 months in acute ATLL and 10 months in lymphoma ATLL. There was no significant difference in the observed cumulative survival according to aggressive ATLL sub-type $(p=0.5)$ (Figure 1). Observed 3- and 5-year survival rates were 18\% and $18 \%$ in acute ATLL and $20 \%$ and $16 \%$ in lymphoma ATLL.

Thirty-two patients received a wide range of second line therapies following progression on first line therapy $(n=15)$ or at relapse $(n=17)$. Responses were seen in 13 (41\%; 4 CR, $9 \mathrm{PR})$. Sixteen patients received $3^{\text {rd }}$ line therapy with partial response in three $(19 \%)$.

Death was due to progressive disease in 56 subjects (92\%), one of whom also had infection, infection without progressive disease in 4 subjects and cardiac failure in one case. Infectious deaths were secondary to CMV pneumonitis, PCP pneumonia and bacterial sepsis (2). Five out of six subjects treated with alloSCT remain alive with median OS 26 months (12-38).

A significant benefit in cumulative survival was demonstrated in subjects with aggressive ATLL treated with AVT at any time $(p<0.0001)$ (Figure 2A). No patient survived 5 years if treated with chemotherapy alone compared with $26 \% 5$-year survival in those treated with AVT at any time. This benefit was demonstrated both in acute $(p=0.0007)$ (Figure 2B) and lymphoma ATLL $(p=0.0004)$ (Figure 2C). Median OS for all with CNS disease at presentation $(n=13)$ was 10.5 months. Seven 
(54\%) were given AVT, as 'combined first line therapy' in three and 'deferred' in four. In this subset the median OS with AVT at anytime was 21.5 months compared with 2.3 months for CNS disease treated without AVT.

Multivariate analysis identified 'use of AVT at any time' as the only factor to reduce risk of death ( $p=0.002$; hazard ratio $0.23,95 \% \mathrm{Cl} 0.091-0.60)$. Other factors included in the analysis were sex, age, disease subtype, LDH, Calcium, lymphocyte count at presentation, bone marrow (BM), liver or spleen involvement.

Timing of AVT was then assessed. While 'combined first line therapy', when compared to chemotherapy alone, was associated with prolonged cumulative OS in both acute $(p=0.0081)$ (Figure 3A) and lymphoma $(p=0.001)$ (Figure 3B) ATLL, AVT was also effective when used after relapse ('deferred therapy'). Of those patients treated with chemotherapy alone first line 14 received ZDV/IFN after relapse with median OS 20 months compared to 4 months if not given at any time $(p=0.002)$. This benefit was demonstrable in acute (median OS 20 months v 4 months; $p=0.03$ ) and lymphoma (median OS 18 v 5.5 months; $p=0.03$ ) ATLL. Furthermore AVT, used either as 'combined first line therapy' or 'deferred therapy', was associated with a comparable benefit in overall cumulative survival compared to chemotherapy alone $(p=0.0006)$ (Figure 4).

\section{Discussion}

Although retrospective, this analysis provides a timely assessment of the diagnosis and treatment of 'aggressive' ATLL in England. A median age at diagnosis of 58 years, while higher than initial reports of ATLL in England and the Caribbean $(11,15)$ is comparable both to more recent UK reports $(16,17)$ and to Japanese data $(12)$. Disease is more common in females in common with all previous reports of ATLL in the UK and the Caribbean and in contrast with Japan where ATLL is more frequent in males (12). Almost twice as many cases of aggressive ATLL were lymphoma, in contrast to the original Shimoyama cohort (8) and previous British studies where acute ATLL was more frequent (15-17) and to a 2010 meta-analysis in which the ratio was approximately $1: 1(22)$.

We have demonstrated that inclusion of ZDV/IFN- $\alpha$, both in 'combined first-line therapy' and in 'deferred therapy' in aggressive ATLL is associated with improved 
survival compared to chemotherapy alone. These data support the recent retrospective meta-analysis, where the use of ZDV/IFN- $\alpha$ was shown to be beneficial in acute ATLL, whilst adding evidence of benefit in lymphoma ATLL.

In acute ATLL our response rate for chemotherapy with AVT is $58 \%$ with a CR rate of $42 \%$. Chemotherapy alone was inferior with a CR rate of $31 \%$ comparable to the $30 \% \mathrm{CR}$ rate with AVT alone in the recent meta-analysis (22). With the exception of the LSG-15 regimen (23), increased response rates following chemotherapy intensification have not been accompanied by significantly improved survival (24). In our study three out of 12 patients treated with combined first line therapy remained alive after median 22 months follow up while no patient treated with chemotherapy alone survived more than 12 months. However two of these three received alloSCT in first remission. Thus, although we demonstrate superiority of combined first line therapy over chemotherapy alone, the impact on survival remains disappointing, and alloSCT should be considered in all cases where satisfactory overall response has been attained, ideally in the context of a clinical trial. We do not attempt to compare with the 'AVT alone' approach as discussed in the meta-analysis where achievement of $\mathrm{CR}$ was associated with a significant benefit in survival. It should be noted that the typical doses of ZDV (1000mg per day) and IFN- $\alpha$ (10 MU daily) are higher when AVT used alone (19).

In ATLL lymphoma_we observed an ORR of $100 \%$ with 'combined first-line therapy' of which $36 \%$ were CR compared with $54 \%$ ORR with chemotherapy alone although the CR rate was similar (39\%). In the meta-analysis, while AVT alone was associated with a survival disadvantage compared with chemotherapy with or without maintenance AVT (median OS 7 months, no patient surviving beyond 18 months), an analysis of the impact of AVT combined with chemotherapy in first line therapy was not made. In our study $6 \%$ of patients treated with chemotherapy alone remained alive at 3 years whilst with 'combined first-line therapy' $27 \%$ survived 3 years with no additional mortality predicted at 6 years. These data would suggest that a combined therapy approach is superior to chemotherapy alone in ATLL lymphoma. In efforts to optimize the chemotherapy alone approach the LSG 15 study group in Japan demonstrated improved CR rates with intensification of chemotherapy compared with biweekly CHOP (40\% v $25 \%$ ) although ORR remained 
around the $70 \%$ mark. This approach resulted in a median OS of 13 months with a 3year OS of $24 \%$ which is comparable to results observed in our study without recourse to chemotherapy intensification. No studies have been performed to test the combination of higher intensity schedules with AVT and this may not be feasible due to toxicity concerns.

The optimal timing for administration of AVT in aggressive ATLL remains to be determined. The observation that survival curves for patients treated with 'combined first-line therapy' or 'deferred therapy' are almost identical suggests that there is no difference and AVT could be deferred to relapse. Two factors argue against such an approach. Firstly response rates are improved by 'combined first-line therapy' over 'chemotherapy alone' and therefore more patients treated with such an approach could be considered for potentially curative alloSCT if eligible. Secondly patients receiving 'deferred AVT' in our study may be biased towards better outcome as they have already survived to the point where second line therapy is considered. Finally, in a recent phase two study evaluating use of AVT after response to first line chemotherapy, only eight out of 19 patients were eligible for AVT, the others having progressed or being withdrawn through toxicity (25).

Although not the subject of this analysis the low rates of Ol support the widespread use of antimicrobial prophylaxis. In addition, since CNS disease was frequent (18\%) early CSF analysis and administration of prophylactic chemotherapy is also recommended. The use of AVT, which penetrates CSF, seems to provide additional benefit.

Despite these results the survival of patients with aggressive ATLL remains poor. Whilst chemotherapy resistance is frequent and well documented it is clear from our results and those from the recent meta-analysis, which together provide survival data on 145 patients with aggressive ATLL treated with AVT, that prognostic indicators to guide therapy would inform therapeutic decision-making. The presence of wild type p53 is reported to influence the response to ZDV (26) whilst expression of IRF4 modifies response to IFN- $\alpha$ (27). Further analysis of the predictive value of these and other markers should be a priority in future studies. 
The heterogeneity of the patients in this study in terms of prior treatment and time to initiating AVT is recognized. Being a retrospective analysis there is always concern that channelling bias of patients with milder or more responsive disease towards AVT may occur. We have attempted to address this by the multivariate analysis and by exploring differences in the timing of ZDV/IFN. We have no evidence that this was driven by patient characteristics but was influenced by local practice. Thus some centres did not start ZDV/IFN at all, some as combined first line therapy and others at relapse.

On the basis of these data we recommend that low dose AVT should be added when chemotherapy is used first line in treatment of both aggressive forms of ATLL. Our data particularly support this approach in lymphoma ATLL. A prospective evaluation of ZDV/IFN- $\alpha$ alone versus 'Combined first line therapy' in treatment of acute ATLL is necessary before ZDV/IFN- $\alpha$ alone can be considered as standard therapy. A detailed analysis of response and survival according to the degree of nodal and/or tissue involvement may prove valuable in identifying those patients most likely to benefit from this treatment. Furthermore this may allow refinement of the diagnostic criteria of ATLL. Although the optimal timing of first line ZDV/IFN- $\alpha$ remains to be confirmed, the increase in ORR afforded by this approach provides a strong platform for a consolidative phase of treatment such as alloSCT if a suitable donor is available. A prospective study would inform further on response rates and toxicity, in order to determine the ideal timing of ZDV/IFN- $\alpha$, as well as providing data on alloSCT as a consolidative therapy. In patients not suitable or eligible for transplantation, but responsive to ZDV/IFN- $\alpha$, maintenance therapy may offer longterm disease control. Finally emerging results with novel agents such as Arsenic trioxide $(28,29)$ and KW-0761, a monoclonal anti-CCR4 antibody (30), are encouraging and may offer alternative post induction strategies. 


\section{REFERENCES}

1. Uchiyama T, Yodoi J, Sagawa K, et al.: Adult T-cell leukemia: clinical and hematologic features of 16 cases. Blood 50:481-492, 1977

2. Yoshida M, Miyoshi I, Hinuma Y: A retrovirus from human leukemia cell lines: its isolation, characterization, and implication in human adult T-cell leukemia (ATL). Princess Takamatsu Symp 12:285-294, 1982

3. Tosswill JH, Taylor GP, Tedder RS, et al.: HTLV-I/II associated disease in England and Wales, 1993-7: retrospective review of serology requests. BMJ 320:611-612, 2000

4. Hanchard B: Adult T-cell leukemia/lymphoma in Jamaica: 1986-1995. J Acquir Immune Defic Syndr Hum Retrovirol 13 Suppl 1:S20-S25, 1996

5. Tajima K: The 4th nation-wide study of adult T-cell leukemia/lymphoma (ATL) in Japan: estimates of risk of ATL and its geographical and clinical features. The T- and B-cell Malignancy Study Group. Int J Cancer 45:237-243, 1990

6. Murphy EL, Hanchard B, Figueroa JP, et al.: Modelling the risk of adult T-cell leukemia/lymphoma in persons infected with human T-lymphotropic virus type I. Int J Cancer 43:250-253, 1989

7. Iwanaga $M$, Watanabe $T$, Utsunomiya $A$, et al.: Human T-cell leukemia virus type I (HTLV-1) proviral load and disease progression in asymptomatic HTLV-1 carriers: a nationwide prospective study in Japan. Blood 116:1211-1219, 2010

8. Shimoyama M: Diagnostic criteria and classification of clinical subtypes of adult T-cell leukaemia-lymphoma. A report from the Lymphoma Study Group (1984-87). Br J Haematol 79:428-437, 1991

9. Dougan S, Payne LJ, Tosswill JH, et al.: HTLV infection in England and Wales in 2002--results from an enhanced national surveillance system. Commun Dis Public Health 7:207-211, 2004

10. Gibbs WN, Lofters WS, Campbell M, et al.: Non-Hodgkin lymphoma in Jamaica and its relation to adult T-cell leukemia-lymphoma. Ann Intern Med 106:361-368, 1987

11. Deardren C, Corbin D, Prussia P, et al.: Clinical and laboratory features of adult T-cell leukaemia lymphoma in Barbados. Leuk Lymphoma 23:561-565, 1996

12. Takatsuki K: Adult T-cell leukemia. Intern Med 34:947-952, 1995 
13. Takatsuki $\mathrm{K}$, Matsuoka M, Yamaguchi K: Adult T-cell leukemia in Japan. J Acquir Immune Defic Syndr Hum Retrovirol 13 Suppl 1:S15-S19, 1996

14. Catovsky D, Greaves MF, Rose M, et al.: Adult T-cell lymphoma-leukaemia in Blacks from the West Indies. Lancet 1:639-643, 1982

15. Matutes E, Catovsky D: ATL of Caribbean origin, in Takatsuki K (ed): Adult Tcell leukaemia. Oxford, Oxford University Press, 1994, pp 113-138

16. Pawson R, Richardson DS, Pagliuca A, et al.: Adult T-cell leukemia/lymphoma in London: clinical experience of 21 cases. Leuk Lymphoma 31:177-185, 1998

17. Matutes E, Taylor GP, Cavenagh J, et al.: Interferon alpha and zidovudine therapy in adult T-cell leukaemia lymphoma: response and outcome in 15 patients. Br J Haematol 113:779-784, 2001

18. Simpson $\mathrm{S}$, Thanigaikumar $\mathrm{M}$, Taylor $\mathrm{G}$, et al.: Combination chemotherapy with CHOP-Daclizumab in HTLV-1 associated adult T-cell leukaemia/lymphoma. Blood 108:2750, 2006 (abstr)

19. Gill PS, Harrington W, Jr., Kaplan MH, et al:: Treatment of adult T-cell leukemia-lymphoma with a combination of interferon alfa and zidovudine. N Engl J Med 332:1744-1748, 1995

20. Hermine O, Bouscary D, Gessain A, et al.: Brief report: treatment of adult Tcell leukemia-lymphoma with zidovudine and interferon alfa. N Engl J Med 332:1749-1751, 1995

21. Yamada $\mathrm{Y}$, Tomonaga $\mathrm{M}$, Fukuda $\mathrm{H}$, et al.: A new G-CSF-supported combination chemotherapy, LSG15, for adult T-cell leukaemialymphoma: Japan Clinical Oncology Group Study 9303. Br J Haematol 113:375-382, 2001

22. Bazarbachi A, Plumelle $\mathrm{Y}$, Carlos RJ, et al:: Meta-analysis on the use of zidovudine and interferon-alfa in adult T-cell leukemia/lymphoma showing improved survival in the leukemic subtypes. J Clin Oncol 28:4177-4183, 2010

23. Tsukasaki K, Utsunomiya A, Fukuda $\mathrm{H}$, et al.: VCAP-AMP-VECP compared with biweekly CHOP for adult T-cell leukemia-lymphoma: Japan Clinical Oncology Group Study JCOG9801. J Clin Oncol 25:5458-5464, 2007

24. Taylor GP, Matsuoka M: Natural history of adult T-cell leukemia/lymphoma and approaches to therapy. Oncogene 24:6047-6057, 2005

25. Ratner $L$, Harrington $W$, Feng $X$, et al.: Human $T$ cell leukemia virus reactivation with progression of adult T-cell leukemia-lymphoma. Plos ONE 4:e4420, 2009 
26. Datta A, Bellon $M$, Sinha-Datta $U$, et al.: Persistent inhibition of telomerase reprograms adult T-cell leukemia to p53-dependent senescence. Blood 108:1021-1029, 2006

27. Ramos JC, Ruiz P, Jr., Ratner L, et al.: IRF-4 and c-Rel expression in antiviral-resistant adult T-cell leukemia/lymphoma. Blood 109:30603068, 2007

28. Ishitsuka K, Suzumiya J, Aoki M, et al.: Therapeutic potential of arsenic trioxide with or without interferon-alpha for relapsed/refractory adult Tcell leukemia/lymphoma. Haematologica 92:719-720, 2007

29. Kchour G, Tarhini M, Kooshyar MM, et al.: Phase 2 study of the efficacy and safety of the combination of arsenic trioxide, interferon alpha, and zidovudine in newly diagnosed chronic adult T-cell leukemia/lymphoma (ATL). Blood 113:6528-6532, 2009

30. Yamamoto K, Utsunomiya A, Tobinai K, et al:: Phase I study of KW-0761, a defucosylated humanized anti-CCR4 antibody, in relapsed patients with adult T-cell leukemia-lymphoma and peripheral T-cell lymphoma. J Clin Oncol 28:1591-1598, 2010

Tables and Figures

Table 1 - Patient characteristics

Table 2 - Response to first line therapy

Figure 1 - Kaplan Meier survival analysis according to ATLL subtype

Figure 2 - Kaplan Meier survival analysis - AVT at any time versus never. (A) All aggressive ATLL; (B) Acute ATLL only; (C) Lymphoma ATLL only.

Figure 3 - Kaplan Meier survival analysis - Combined first line therapy versus chemotherapy alone. (A) Acute ATLL; (B) Lymphoma ATLL.

Figure 4 - Kaplan Meier survival analysis - Aggressive ATLL treated with 'Combined first line therapy' versus 'Deferred therapy' versus 'Chemotherapy alone' 


\begin{tabular}{|c|c|c|c|}
\hline & All & Acute & Lymphoma \\
\hline Female, $\mathrm{n}(\%)$ & $44(60)$ & $19(66)$ & $25(57)$ \\
\hline Age (years) - Median & & & \\
\hline - Range & 58 & & \\
\hline ECOG Performance Status & $22-85$ & 55 & $22-85$ \\
\hline (Median) & 1 & $37-771$ & 1 \\
\hline Splenomegaly & $25 \%$ & $34 \%$ & $18 \%$ \\
\hline Liver involvement & $22 \%$ & $38 \%$ & $11 \%$ \\
\hline Bone marrow involvement & $29 \%$ & $59 \%$ & $30 \%$ \\
\hline CNS involvement & $18 \%$ & $17 \%$ & $18 \%$ \\
\hline Lymphocytes (x109\%/L) & & & \\
\hline (median) & 3.3 & 14.3 & 2.0 \\
\hline $\mathrm{Hb}(\mathrm{g} / \mathrm{dL})$ (median) & 12.8 & 12.9 & 12.5 \\
\hline Platelets (x109/L) (median) & 268 & 273 & 259 \\
\hline Hypercalcaemia, (\%) & $42 \%$ & $62 \%$ & $28 \%$ \\
\hline LDH >2xULN, $(\%)$ & $69 \%$ & $82 \%$ & $61 \%$ \\
\hline
\end{tabular}

\section{Table 1}




\begin{tabular}{|c|l|c|c|c|c|c|}
\hline & Treatment & $\mathrm{N}$ & ORR & CR & PR & SD/PD \\
\hline All & $\begin{array}{l}\text { Chemotherapy } \\
\text { alone }\end{array}$ & 39 & $49 \%$ & $36 \%$ & $13 \%$ & $51 \%$ \\
\hline $\begin{array}{l}\text { Combined first line } \\
\text { therapy }\end{array}$ & 26 & $81 \%$ & $39 \%$ & $42 \%$ & $19 \%$ \\
\hline Acute ATLL & $\begin{array}{l}\text { Chemotherapy } \\
\text { alone }\end{array}$ & 13 & $39 \%$ & $31 \%$ & $8 \%$ & $62 \%$ \\
\hline Combined first line \\
therapy
\end{tabular}

Table 2 


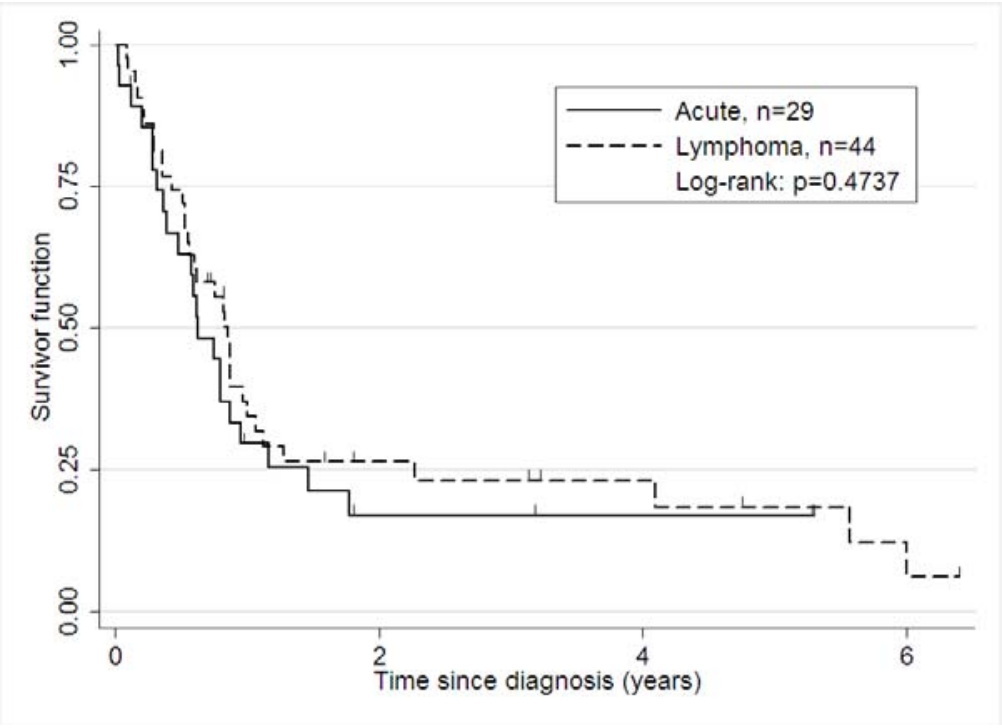

\section{Figure 1}

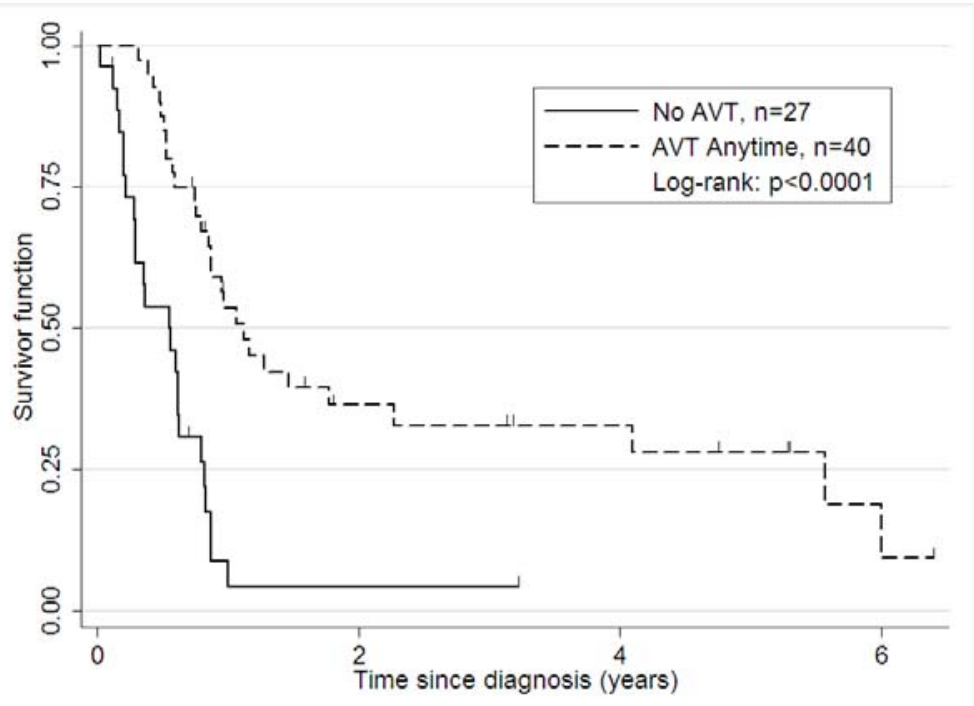

Figure 2 (A) 


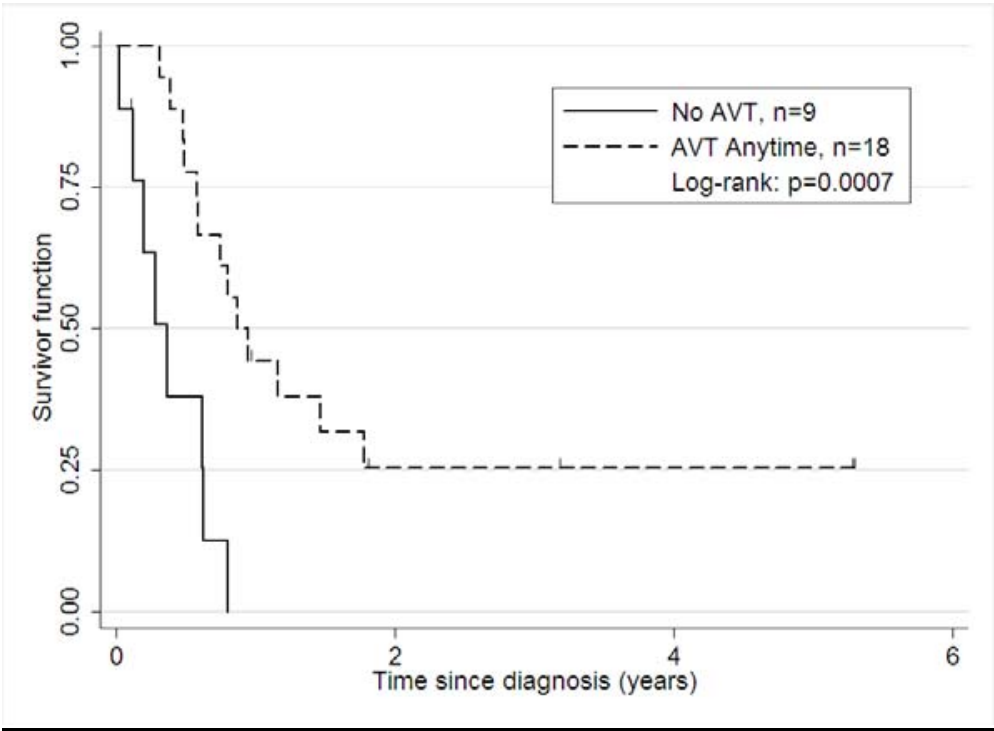

\section{Figure 2(B)}

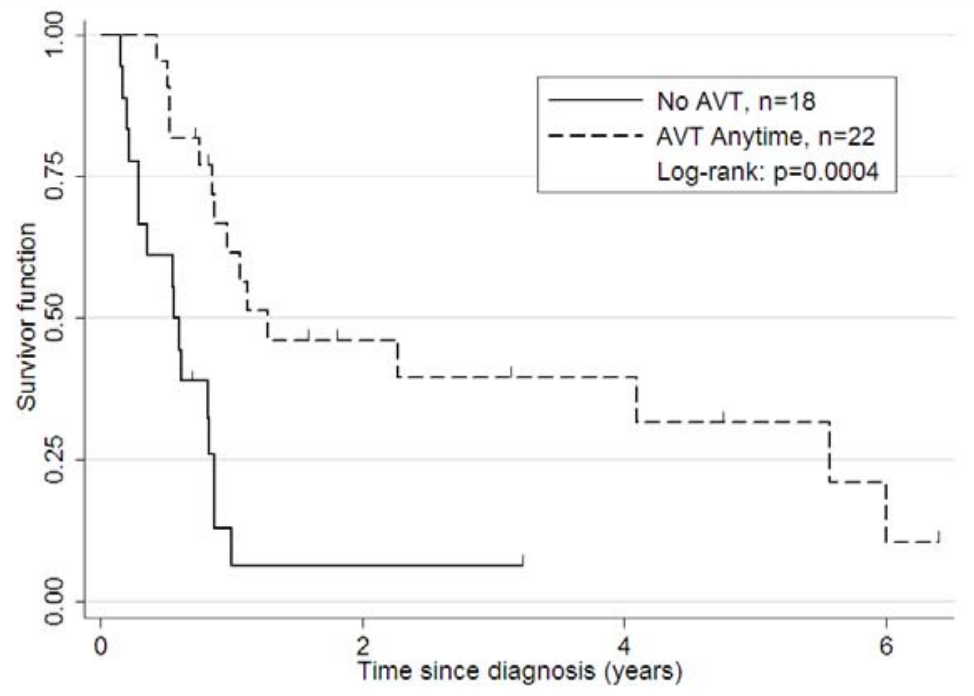

Figure 2 (C) 


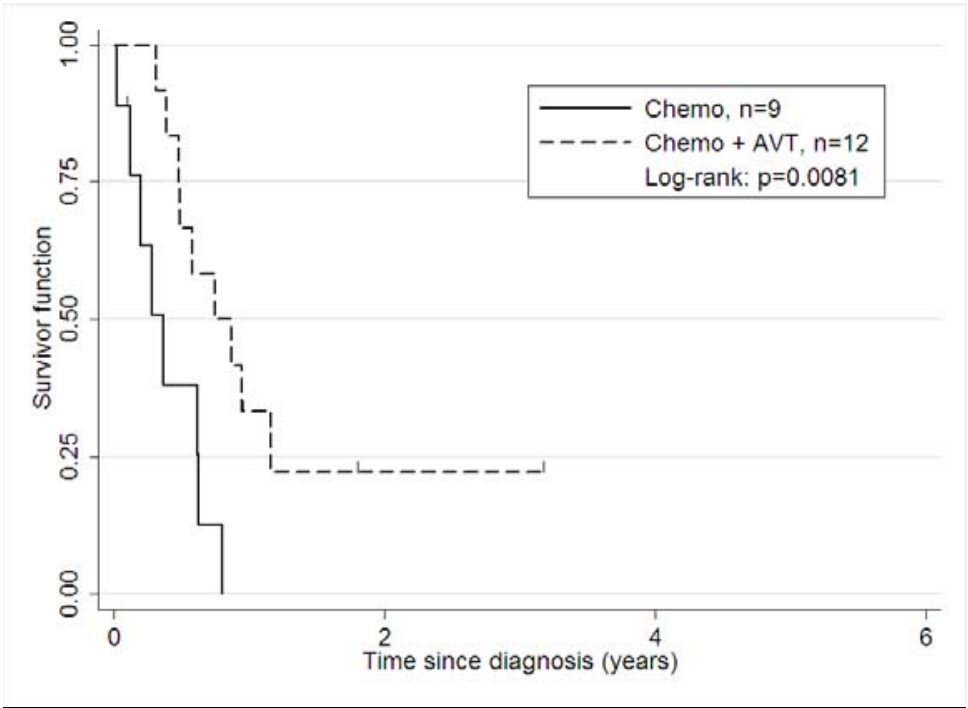

\section{Figure $3(\mathrm{~A})$}

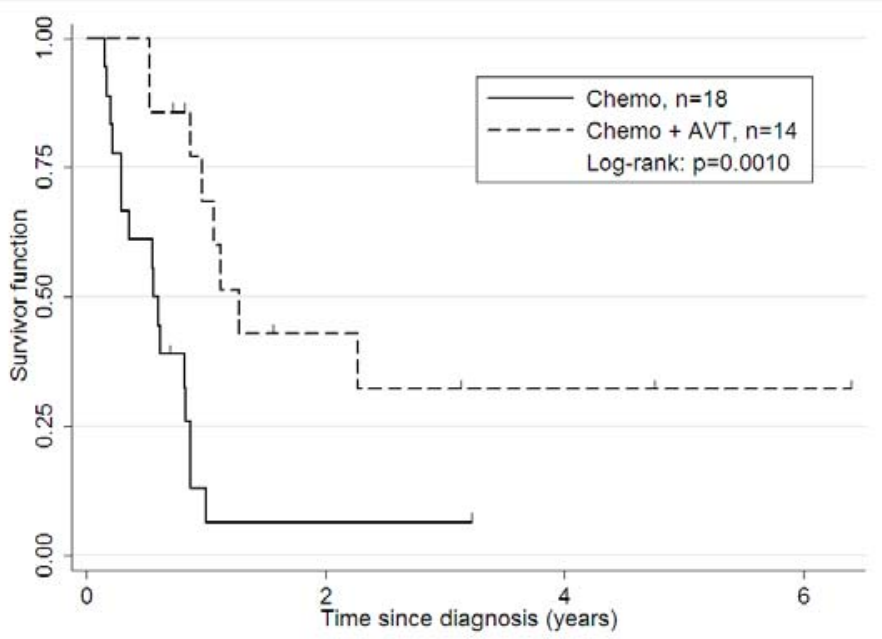

Figure 3 (B) 


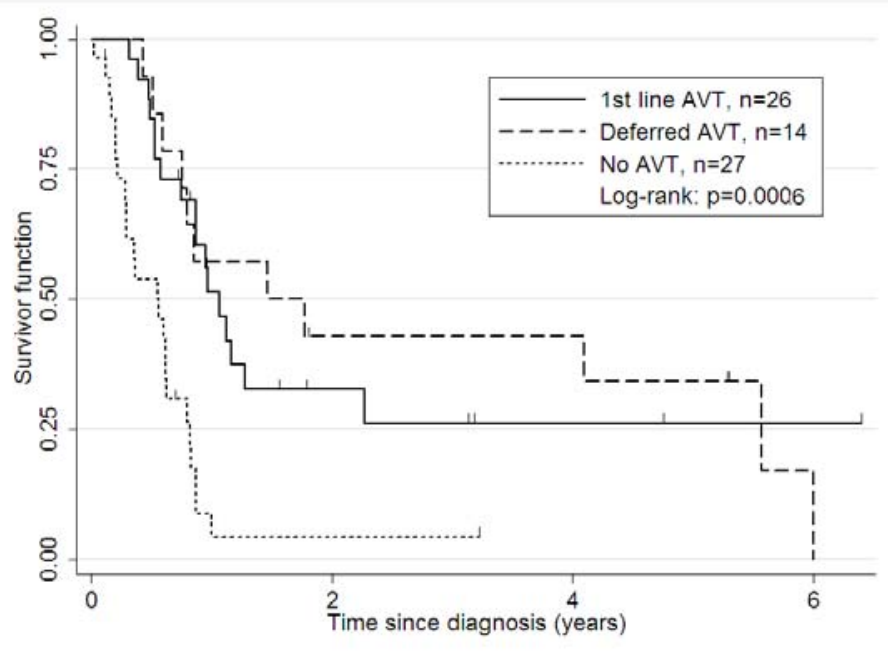

Figure 4 